\title{
Research on Mathematical Understanding in Mainland China
}

\author{
Zhao Kai, Yang Zezhong*, Sun Dandan \\ School of mathematics science, Shandong Normal University, Jinan, China \\ Email: zhongzee@163.com
}

\begin{abstract}
Mathematical understanding has been a hot topic of mathematical research in mainland China. Over the years, many researchers studied characteristics, process, level and evaluation of mathematical understanding with different methods and achieved many results. Many researchers think that the process of mathematical understanding is a process in which mathematical knowledge is assimilated and adapted, and the level of mathematical understanding is progressive approached. However, there are still some shortcomings in researches on characteristics and evaluation.
\end{abstract}

Keywords-Mathematical Understanding; Mathematics Learning; Mathematics Teaching

\section{INTRODUCTION}

Mathematical understanding is an important part of the mathematics learning. Many Chinese researchers think it is the key of mastering and applying mathematical knowledge. Lv Linhai believes that the mathematical understanding can improve the students' thinking ability and the ability of solving practical problems [1]. Zhang Wenhui and Wang Guangming think that mathematical understanding can help the students to learning the new knowledge [2]. Pi Lei and Cui Hongyu think that mathematical understanding can help students to master the essence of mathematical knowledge [3]. Yang Zezhong thinks that the mathematical understanding can directly serve the practical mathematics teaching, thus it has great significance for the mathematics practice [4]. Zhang Chunguang believes that the understanding of mathematical concept can improve the ability of learning new knowledge [5]. Niu Teng believes the understanding of mathematical concept can help students to master mathematical knowledge and improve the mathematical quality [6]. So there are lots of research results about mathematical understanding in mainland China. This paper is to summarize these researches.

\section{CHARACTERISTICS OF MATHEMATICAL}

\section{UNDERSTANDING}

Lv Linhai thinks that mathematical understanding is generative and developmental. What "generative" means is that all the complicated mathematical concepts and propositions can be understood in a different way; the so-called "developmental" refers to the use and connection of the knowledge to promote the further development of understanding [1]. Bi Ligetu, Shi
Ningzhong and Ma Yunpeng argue that the mathematical understanding is subjective, different and cyclical. What "subjective" means is that mathematical understanding must have the subject; the so-called "different" refers to due to the different ages, traditions and backgrounds, there are some differences between today's understanding and the traditional understanding; what "cyclical" means is that understanding is a never-ending dynamic cycle process[7]. Wang Ruilin and Qi Chun believe that the mathematical understanding is procedural, silent and hierarchical. The so-called "procedural" refers to the process that mathematical understanding is the new knowledge coming into the original cognitive structure; what "silent" means is that the hidden knowledge of mathematics is contained in the mathematical understanding; the so-called "hierarchical" refers to the students have different levels of knowledge construction [8].

\section{PROCESS OF MATHEMATICAL UNDERSTANDING}

$\mathrm{Ma} \mathrm{Fu}$ thinks that the process of mathematical understanding is a process of building understanding of learning object [9]. Yu Xinhua and Yang Zhi argue the process of mathematical understanding is a process of construction. The construction includes absorbing, processing and bringing into of the informati on [10]. Zhang Chunguang thinks that the understanding of mathematical concept is a two-way operation process [5]. Yang Zezhong agrees with Zhang Chunguang's points and thinks that the process of mathematical understanding consists of two parts. One part is the process of "to", the other part is process of "back". In "to" process, students perform the derived learning, establish vertical association, in "back" process, students use the old knowledge to infer new knowledge, establish lateral ties [4]. Niu Teng says the psychological process of mathematical understanding is the process of new concepts contacting old concepts and meanwhile the old concept contacting new concept actively [6].

\section{LEVEL OF MATHEMATICAL UNDERSTANDING}

Tian Wanhai considers that mathematical understanding has three stages: preliminary understanding, precise understanding and deep understanding [11]. Xiang Xianying thinks that the mathematics understanding has hierarchy and it can be divided into five levels: pre-understanding, misunderstanding, inadequate understanding, understanding and after understanding [12]. Liu 
Lianghua thinks mathematical understanding has six dimensions: explanation, self interpretation, application migration, critical insight, empathy experience and self reflection [13]. Lv Linhai divides the mathematical understanding into four levels: empirical understanding, formal understanding, structured understanding and cultural understanding. Yu Xinhua divides the mathematical understanding into five levels: zero level, common sense level, logic level, idea level, and endless level. Yu Xiuhui divides the mathematical understanding into four levels: experiential cognition stage, formal cognition stage, relational cognition stage and idea cognition stage. Zhong Zhihua divides the mathematical understanding into four levels: memorial comprehension, operational understanding, intuitive understanding and analysis understanding. Yang Huiqing divides the mathematical understanding into four levels: operational memory, conceptual memory, interpretive understanding and inquiry understanding [14].

\section{EVALUATION OF MATHEMATICAL UNDERSTANDING}

Lv Linhai believes that we can use three hypotheses to evaluate mathematical understanding: first, evaluation should be viewed as a continuous process; second, evaluation should include classroom communication and written works; third, evaluation should include both informal assessment and formal assessment [1]. Yang Huiqing thinks that the evaluation of mathematical understanding should combine qualitative and quantitative methods - weighted summation method [14]. Cai Jin considers that mathematical understanding should be evaluated by qualitative and quantitative methods [15]. Wang Xinbing believes that we should use multiple tracking methods to get a high reliability and validity of the results [16].

\section{RETHINKING}

Although the Chinese researchers have made quite a few valuable and got significant results in mathematical understanding at present, after reviewing these researches, the author thinks Chinese researchers still need further efforts, especially in the following aspects:

1. The process of mathematical understanding. At present, many researchers have been aware of that the process of mathematical understanding is related to the internal cognitive structure, and it is a process of the assimilation and adaptation as a whole. But further researches about process of mathematical understanding obviously are still not many yet now, particularly the detail and explicit researches which can guide the mathematics teaching directly are very few. However, this kind of researches is quite necessary, so the author hopes there will be more researches about process of mathematical understanding.

2. The level of mathematical understanding. There are many results in this aspect and many of them are different expressions of the surface but the meaning is same, the domestic researchers' perspective is that the level of mathematical understanding is progressive approached. There are also some problems in this field: first of all, the researchers use the previous research ideas and methods, and the innovation are seriously inadequate. Secondly, the results have not given the specific performance characteristic which makes it difficult for us to define which level students are in. I hope that the researchers can use the new research ideas, but do not rigidly adhere to the existing research results.

3 . The characteristics of mathematical understanding. There is little research in this aspect and through summing up the domestic researches, the characteristics of mathematical understanding are generative, procedural, silent, developmental, complex, universal and cycle. But there are also some shortcomings. For example, the same nature has different explanation, for example, Lv Linhai and $\mathrm{Li}$ Shaobao think the mathematical understanding is developmental, but it can be seen that the explanations of development are different.

4. The evaluation of mathematical understanding. There is little research and discussion in this aspect, the main point of domestic researchers is we can not use a single evaluation and should use a comprehensive evaluation, such as taking the combination of qualitative and quantitative. The author thinks that the biggest problem is that it is not given a strict evaluation standard, which makes it very difficult for us to evaluate the students. Therefore, the author hopes that the researchers are able to work on these aspects.

\section{REFERENCES}

[1]Lv, L. H., The View of Mathematical Understanding. Reference of Math Teaching in Middle School, 32 (12) (2003) 1-4.

[2]Zhang, W. H. \&Wang, G. M., Review on Mathematical Cognitive Understanding. Journal of Qufu Normal University, 31 (1) (2005) 120-124.

[3]Pi, L. \& Cui, H. Y., Mathematics Understanding and Understanding Memorization. Journal of Sichuan College of Education, 24 (5) (2008) 109-110.

[4]Yang, Z. Z., Investigation of the Process of Mathematics Understanding. Journal of Mathematics Education, 21 (6) (2012) 50-53.

[5]Zhang, C. G., Cognitive Process of Understanding Mathematical Concepts (Unpublished master dissertation). Shandong Normal University, Jinan, (2011).

[6]Niu, T., The Psychological Process Research of Junior School Students during Deep Understanding of Mathematical Concepts (Unpublished master dissertation). Shandong Normal University, Jinan, (2013).

[7]Bi, L. G. T., Ma Y. P. \& Shi, N. Z., The Understanding of "Understanding" Based on the View of Mathematics. Journal of Northeast Normal University (Philosophy and Social Sciences), 61 (2) (2011) 159-163.

[8]Wang, R. L. \& Qi, C., The five layers of mathematics understanding and teaching strategies. Course Education Research, 14 (5) (2012) 46-48.

[9]Ma, F., On Two Types of understanding in Mathematics. Journal of Mathematics Education, 10 (3) (2001) 50-53.

[10]Yu, X. H. \& Yang, Z., Made and Application of Higher Mathematics Multi-media Teaching Plan. Journal of Mathematics Education, 14 (2) (2005) 23-26.

[11]Tian, W. H., Mathematics Pedagogy. Zhejiang Education Publishing House, Hangzhou, 1993, pp. 88-89.

[12]Xiang, X. Y., Levels of Mathematics Understanding. Journal of Suzhou University, 22 (5) (2007) 97-100.

[13]Liu, L. H., Six Kinds of Dimension of Mathematics Understanding and Its Enlightenment. Journal of Nei Mongo Normal University (Educational Science), 22 (6) (2009) 132-135.

[14]Yang, H. Q., On the Evaluation Method for Mathematical 
Understanding and Mathematical Model Construction. Journal of Chuzhou University, 14 (5) (2012) 10-13.

[15]Cai, J., Analysis of Qualitative and Quantitative Methods for Evaluating the Level of Mathematical Understanding. Journal of
Changchun Education Institute, 29 (18) (2013) 75-77.

[16]Wang, X. B., A Comment on Understanding of Mathematics Learning. Journal of Mathematics Education, 17 (5) (2008) 94-96. 